\title{
Chylous Ascites in a Patient with Inflammatory Myofibroblastic Tumor
}

\author{
Sascha Dietrich Gerlinde Egerer Anthony D. Ho \\ Bernd Kasper \\ Department of Internal Medicine V, University of Heidelberg, Heidelberg, \\ Germany
}

\section{Key Words}

Inflammatory myofibroblastic tumor · Chyloperitoneum - Chylous ascites · Octreotide therapy

\begin{abstract}
Background: We present the case of a 64-year-old patient who presented to his primary care physician with fatigue, worsening shortness of breath, abdominal discomfort and a rapidly growing abdominal girth, although he had lost $5 \mathrm{~kg}$ of weight within 3 months. He had a history of untreated hypertension, compensated renal insufficiency and COPD. Despite weight loss and fatigue, the patient did not experience any other constitutional symptoms such as fever, night sweats or loss of appetite.

Investigations: Physical examination, blood tests, CT scan of the abdomen, MRI scan of the abdomen, fine needle biopsy, excisional biopsy, Video Capsule Endoscopy, histology, PET scan.

Diagnosis: Inflammatory myofibroblastic tumor, chylous ascites, chyloperitoneum. Management: Systemic chemotherapy, total parenteral nutrition and octreotide therapy.

Conclusion: We describe the case of a patient in whom two extremely rare phenomena are present in combination: the diagnosis of an inflammatory myofibroblastic tumor and chylous ascites. While the tumor could be stabilized by different regimens of chemotherapy, the chyloperitoneum was treated with parenteral nutrition and subcutaneous octreotide injections, which resulted in a significant reduction of the amount of chylous ascites drained during regular paracentesis.
\end{abstract}

\section{Case Report}

We report a 64-year-old patient who presented to his primary care physician with fatigue, worsening shortness of breath, abdominal discomfort and a rapidly growing abdominal girth. The patient had lost $5 \mathrm{~kg}$ of weight within the last 3 months. He had been well until 3 months prior to his first visit at his 
primary care physician. Furthermore, the patient had a history of COPD, hypertension and compensated renal insufficiency.

A CT as well as a subsequent MRI scan (fig. 1) revealed a large tumor of the upper left abdomen infiltrating the entire mesentery and the omentum majus. The tumor was up to $15 \mathrm{~cm}$ in diameter and ascites could be found radiologically. A fine needle biopsy of the abdominal mass was performed at another hospital, but not enough tissue could be obtained for a diagnosis to be made. Subsequently, the patient underwent a laparoscopy for biopsy, which confirmed the diagnosis of an inflammatory myofibroblastic tumor. During the procedure, a cloudy and milky fluid could be drained, which was likely to be chylous ascites. The milky fluid could be collected in wound drainage for several days after surgery.

The patient was transferred to our medical oncology clinic. On admission, the patient presented with large and distended abdomen. Abdominal ultrasound examination confirmed ascites, and abdominal paracentesis drained 4 liters of milky fluid (fig. 2). Biochemical analysis confirmed the diagnosis of chylous ascites. Total protein content was $46.3 \mathrm{~g} / \mathrm{l}$; glucose was $103 \mathrm{mg} / \mathrm{dl}$. Cholesterol and triglyceride concentrations were 301 and $702 \mathrm{mg} / \mathrm{dl}$, respectively. Serum triglyceride levels were not elevated (159 $\mathrm{mg} / \mathrm{dl}$ ). Cytological examination found 910 cells showing a few activated mesothelial cells, but no tumor cells or signs of infection could be seen.

We presented the case to our Department of Surgery at the University of Heidelberg. Due to the size and the massive tumor infiltration of the mesentery, radical resection was judged to be impossible. Therefore, we decided to treat the patient with a chemotherapy regimen consisting of etoposide 125 $\mathrm{mg} / \mathrm{m}^{2} /$ day administered on day 1 and 4 , ifosfamide $1,500 \mathrm{mg} / \mathrm{m}^{2} /$ day on day $1-4$ and adriamycin 50 $\mathrm{mg} / \mathrm{m}^{2} /$ day on day 1 (EIA regimen, repetition day 22). A MRI scan after 2 cycles of chemotherapy did not show any shrinkage of the tumor and was therefore evaluated as stable disease. In addition, we performed a fluorodeoxyglycose positron emission tomography (PET) scan after these 2 cycles of chemotherapy, which did not reveal a significantly elevated fluorodeoxyglycose uptake of the abdominal tumor (maximal standard uptake value 4.4). This result underlines the tendency of the tumor to grow very slowly. Therefore, we decided together with the patient to discontinue aggressive chemotherapy.

For the treatment of the chylous ascites, the patient was put on a low total parenteral nutrition (TPN). Subcutaneous octreotide therapy (Sandostatin; Novartis Pharmaceuticals, Nurnberg, Germany) was started $(3 \times 50 \mu \mathrm{g}$ per day) after completion of the first cycle. As no significant side effects were noted, the octreotide dose was increased after one week of treatment to $3 \times 100 \mu \mathrm{g}$ per day, and after another week to $3 \times 150 \mu \mathrm{g}$ per day. Total parenteral nutrition was performed simultaneously to octreotide therapy. As a result of this therapeutic approach the amount of chylous ascites, which could be drained during regular paracentesis, was significantly reduced (fig. 3 ). Altogether, total parenteral nutrition and octreotide therapy were discontinued after 6 weeks.

Follow-up assessment 2 months after completion of the first 2 cycles of chemotherapy showed a recurrence of the chyloperitoneum (fig. 3) as well as a mild tumor growth. At this point, we decided to continue with the parental nutrition as an overnight infusion because of the increased risk of permanent malnutrition, which had led to further weight loss of the patient. Because of life quality reasons the patient decided to eat as usual during daytime. Furthermore, we started to treat the patient with another chemotherapy regimen consisting of methotrexate $\left(30 \mathrm{mg} / \mathrm{m}^{2}\right.$ day 1,15 , and 22$)$, vinblastin $\left(3 \mathrm{mg} / \mathrm{m}^{2}\right.$ day 1,15 , and 22$)$, doxorubicin $\left(30 \mathrm{mg} / \mathrm{m}^{2}\right.$ day 2$)$ and cyclophosphamid (200 $\mathrm{mg} / \mathrm{m}^{2}$ day 2$)$, which was repeated once after 28 days. According to Dishop et al., this regimen was effective in inflammatory myofibroblastic tumors [1]. This approach helped to further reduce chylous ascites (fig. 3), but did not result in any tumor shrinkage, which could be proven by a follow-up MRI scan of the abdomen. In search of alternative therapeutic strategies we preformed a DOTA-D-Phe1-Tyr3-octreotide (DOTATOC) positron emission tomography CT, which unfortunately showed no somatostatin receptor expression of the abdominal tumor. Therefore, yttrium-90 labeled DOTATOC therapy was not indicated. The patient was then discharged home, continuing overnight parental nutrition. For 3 months, the patient has been stable at home and paracentesis was necessary only once.

\section{Discussion of Diagnosis}

Inflammatory myofibroblastic tumors (IMT) are extremely rare lesions grouped within the family of fibroinflammatory disorders. Originally, they have been classified as benign. However, some have been shown to demonstrate chromosomal aberrations resulting in 
locally aggressive behavior and rarely metastasis. Therefore, these destructive variants can be counted to the heterogeneous group of soft tissue sarcomas. They may arise in almost any organ system and have already been described in the lung, spleen, bladder, pancreas, liver, colon, peripheral nerves, and soft tissue. IMT have been predominantly found in children and young adults; nevertheless, they can affect all age groups [2]. The characteristic histopathological feature of these lesions is the myofibroblast showing a dominant spindle cell proliferation with a variable inflammatory component [3]. Clinically, these tumor lesions lead to mass effects and patients' discomfort is usually the reason for medical consultation, which was the case in our patient, too [2]. The primary and curative therapeutic approach is surgery if the anatomic location is amenable to radical resection. If an extirpation is not possible, like in the case we have presented here, lesions are observed or treated with various regimens, including non-steroidal antiinflammatory agents, steroids, radiation, and chemotherapeutic drugs [1]. We decided to treat our patient with a chemotherapy regimen consisting of etoposide $125 \mathrm{mg} / \mathrm{m}^{2} / \mathrm{day}$ administered on day 1 and 4 , ifosfamide $1,500 \mathrm{mg} / \mathrm{m}^{2} /$ day on day $1-4$ and adriamycin 50 $\mathrm{mg} / \mathrm{m}^{2} /$ day on day 1 (EIA regimen, repetition day 22). This regimen is well established for the neoadjuvant/adjuvant treatment of soft tissue sarcoma at our institution. MRI after two cycles of chemotherapy showed stable disease according to RECIST criteria. The EIA regimen was not effective to cause any shrinkage of the abdominal tumor. That is why we decided together with the patient to use a different regimen consisting of methotrexate $\left(30 \mathrm{mg} / \mathrm{m}^{2}\right.$ day 1,15 , and 22 ), vinblastin $\left(3 \mathrm{mg} / \mathrm{m}^{2}\right.$ day 1,15 , and 22$)$, doxorubicin (30 $\mathrm{mg} / \mathrm{m}^{2}$ day 2$)$ and cyclophosphamid $\left(200 \mathrm{mg} / \mathrm{m}^{2}\right.$ day 2$)$, which was described to be effective in inflammatory myofibroblastic tumors by Dishop et al. [1]. After 2 cycles, a further MRI scan was performed showing no significant response of the inflammatory myofibroblastic tumor. At this point we decided not to continue chemotherapy because the role of chemotherapy in the treatment of inflammatory myofibroblastic tumors remains questionable. The majority of case reports that have demonstrated a benefit from chemotherapy in the treatment of inflammatory myofibroblastic tumors exists in the pediatric population. There is almost no experience in the treatment with chemotherapy of elderly patients suffering from inflammatory myofibroblastic tumors. In Kovach's review, only 3 patients were treated with systemic chemotherapy, comprising schedules of vincristine, methotrexate, ifosfamide, carboplatin, etoposide and taxol with inconsistent benefits [2]. However, the length and composition of chemotherapy should be based on the aggressiveness and evidence of local invasion of the tumor. This information can be obtained partly by PET. As described we have performed a fluorodeoxyglycose PET scan after the first tow cycles of EIA, which showed no significantly elevated uptake of fluorodeoxyglycose (maximal standard uptake value 4.4). This data underlines the tendency of the tumor to grow very slowly and, therefore, its being insensitive to chemotherapy.

Interestingly, another extremely rare phenomenon could be found in our patient. Abdominal paracentesis revealed cloudy fluid confirmed as chylous ascites which developed due to the massive tumor infiltration of the mesentery and the omentum majus. Chylous ascites is an uncommon finding with an incidence of approximately 1 per 20,000 admissions [4]. Chylous ascites develops when the lymphatic flow between the bowel and the cisterna chyli is disrupted. Commonly, it is observed as a challenging complication of thoracic or abdominal surgery [5-7]. Furthermore, liver cirrhoses, infections or malignancies in particular lymphomas have been reported to cause chylous ascites as well $[6,8,9]$. Although there is no recent study, it is generally believed that the incidence has increased due to the longer survival of patients with cancer and more aggressive abdominal and cardiothoracic interventions. 
There is no standard treatment of chylous ascites. Repeated paracentesis has been used but can induce severe malnutrition and even death. If treatment of the underlying cause of chylous ascites is impossible or success of the treatment is delayed, conservative treatment options involve a medium chain triglyceride-based diet or total parenteral nutrition [10]. However, existing experience is rare and most cases were reported in children. Total parenteral nutrition reduces the accumulation of chylous fluid in the peritoneal cavity, but a satisfactory outcome requires at least 3-4 weeks of treatment [11]. There is anecdotal experience in using the somatostatin analogue octreotide to decrease the intestinal blood flow and inhibit lymph secretion through somatostatin receptors in the intestinal wall, thereby improving chylous ascites. Two cases on successful treatment of chylous ascites with octreotide have been reported in children [11, 12]. Another case report describes the successful use of octreotide in a patient with chylous ascites after liver transplantation [13]. Octreotide represents a promising adjunct in the conservative treatment of chylous ascites. Recent development of a long-acting somatostatin analogue could even facilitate patient's treatment options.

We have put our patient on parenteral nutrition over night to avoid malnutrition. During the day, the patient decided to eat whatever he liked for life quality reasons. In addition, we initially treated the patient with subcutaneous octreotide injections for more than 1 month. This management led to a significant long term reduction of the chylous ascites and, therefore, can be recommended for even those patients where the underlying cause for chylous ascites can not be treated.

Fig. 1. Initial MRI before start of therapy demonstrating a large tumor of the upper left abdomen infiltrating the mesenterium and the omentum majus.

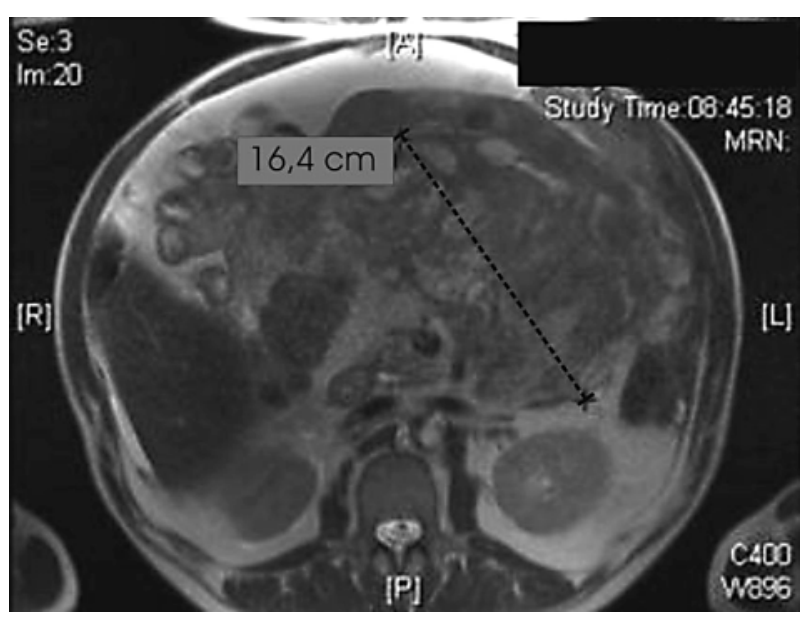




\begin{tabular}{c|l|l|l}
$\begin{array}{c}\text { Cose Reports in } \\
\text { Oncolady }\end{array}$ & $\begin{array}{l}\text { Case Rep Oncol 2009;2:144-149 } \\
\text { D0I: 10.1159/000231972 }\end{array}$ & Published online: August 1, 2009 & $\begin{array}{l}\text { O 2009 S. Karger AG, Basel } \\
\text { ISSN 1662-6575 } \\
\text { www.karger.com/cro }\end{array}$ \\
\hline
\end{tabular}

Fig. 2. Chylous ascites.

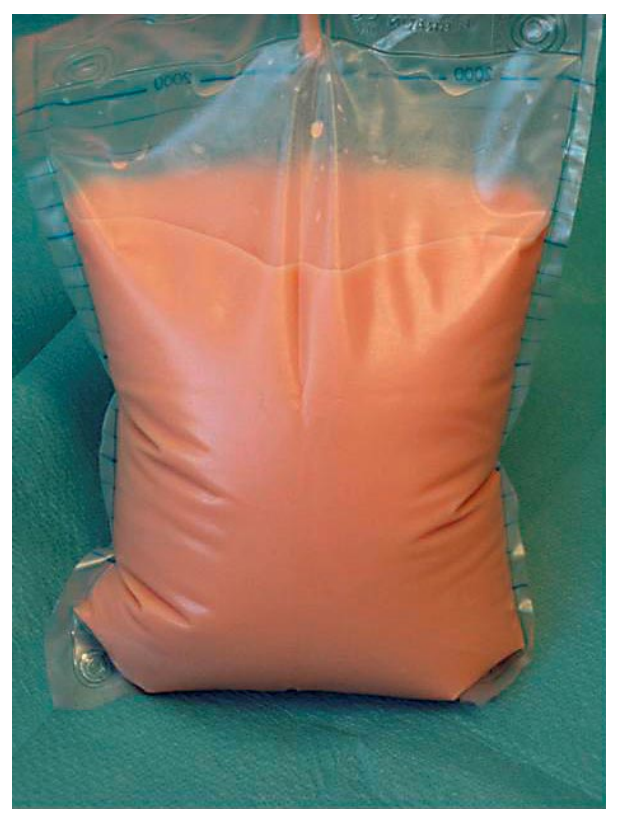

Fig. 3. Reduction of chylous ascites which could be drained during regular paracentesis. a Start of total parenteral nutrition and octreotide therapy. $\mathbf{b}$ Discontinuation of total parenteral nutrition and octreotide therapy. c Continuation of total parenteral nutrition.

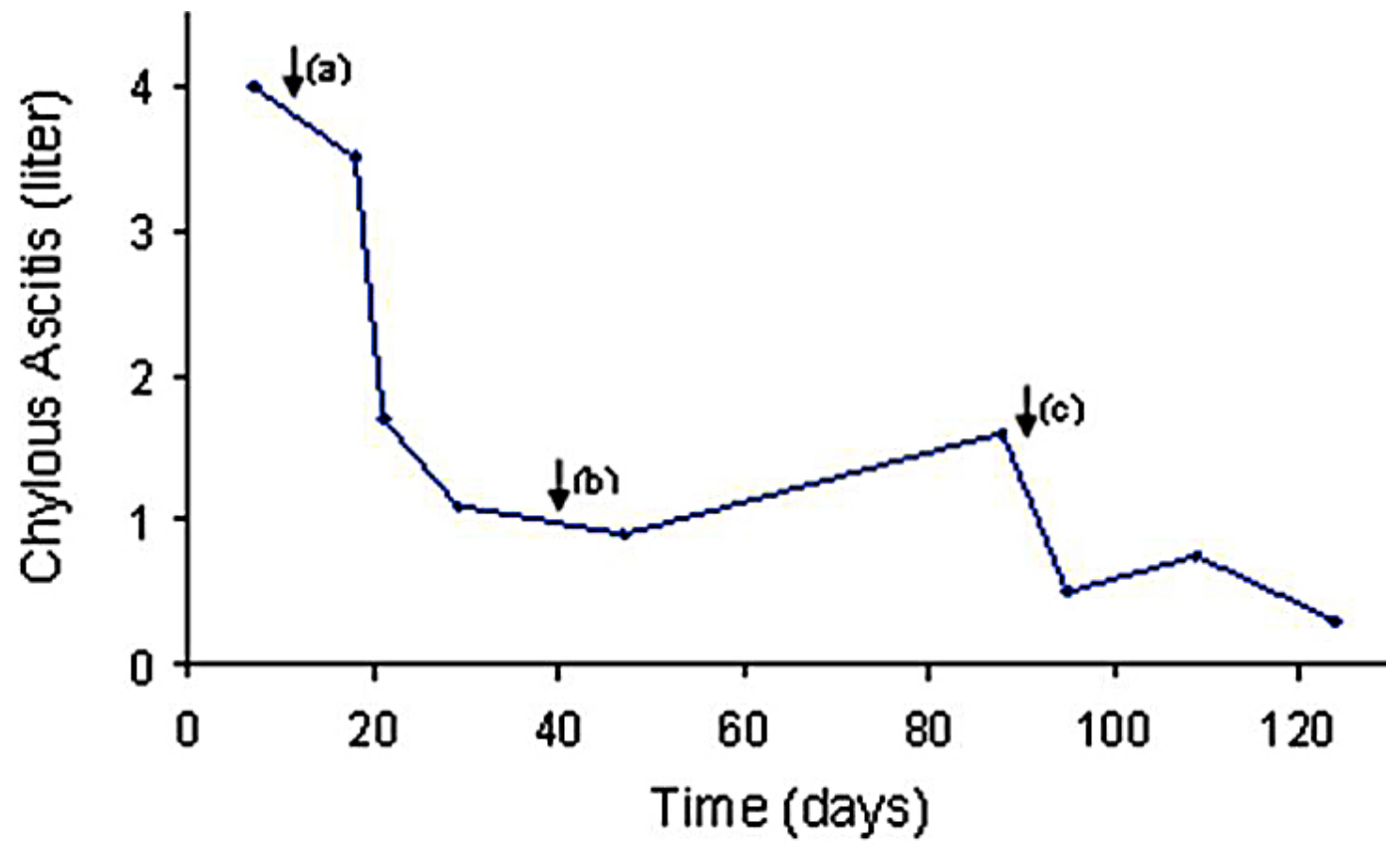




\section{References}

1 Dishop MK, Warner BW, Dehner LP, et al: Successful treatment of inflammatory myofibroblastic tumor with malignant transformation by surgical resection and chemotherapy. J Pediatr Hematol Oncol 2003;25:153-158.

2 Kovach SJ, Fischer AC, Katzman PJ, et al: Inflammatory myofibroblastic tumors. J Surg Oncol 2006;94:385-391.

-3 Coffin CM, Dehner LP, Meis-Kindblom JM: Inflammatory myofibroblastic tumor, inflammatory fibrosarcoma, and related lesions: an historical review with differential diagnostic considerations. Semin Diagn Pathol 1998;15:102-110.

$\checkmark 4$ Press OW, Press NO, Kaufman SD: Evaluation and management of chylous ascites. Ann Intern Med 1982;96:358-364.

5 Gaglio PJ, Leevy CB, Koneru B: Peri-operative chylous ascites. J Med 1996;27:369-376.

6 Romero S, Martin C, Hernandez L, et al: Chylothorax in cirrhosis of the liver: analysis of its frequency and clinical characteristics. Chest 1998;114:154-159.

7 Asfar S, Lowndes R, Wall WJ: Chylous ascites after liver transplantation. Transplantation 1994;58:368-369.

$\checkmark 8$ Browse NL, Wilson NM, Russo F, al-Hassan H, Allen DR: Aetiology and treatment of chylous ascites. Br J Surg 1992;79:1145-1150.

9 Selk A, Wehrli B, Taylor BM: Chylous ascites secondary to small-bowel angiosarcoma. Can J Surg 2004;47:383-384.

10 Weinstein LD, Scanlon GT, Hersh T: Chylous ascites. Management with medium-chain triglycerides and exacerbation by lymphangiography. Am J Dig Dis 1969;14:500-509.

11 Man DW, Spitz L: The management of chylous ascites in children. J Pediatr Surg 1985;20:72-75.

12 Al-Ghamdi MY, Bedi A, Reddy SB, Tanton RT, Peltekian KM: Chylous ascites secondary to pancreatitis: management of an uncommon entity using parenteral nutrition and octreotide. Dig Dis Sci 2007;52:2261-2264.

13 Shapiro AM, Bain VG, Sigalet DL, Kneteman NM: Rapid resolution of chylous ascites after liver transplantation using somatostatin analog and total parenteral nutrition. Transplantation 1996;61:1410-1411. 